\title{
Preflight Adaptation Training for Spatial Orientation and Space Motion Sickness
}

\author{
Deborah L. Harm. PhD, and Donald E. Parker, PhD
}

Two part-task preflight adaptation trainers (PATs) are being developed at the NASA Johnson Space Center to preadapt astronauts to novel sensory stimulus conditions similar to those present in microgravitv to facilitate adaptation to microgravity and readaptation to Earth. This activity is a major component of a general effort to develop countermeasures aimed at minimizing sensorv and sensorimotor disturbances and Space Motion Sickness (SMS) associated with adaptation to microgravity and readaptation to Earth. Design principles for the development of the two trainers are discussed, along with a detailed description of both devices. In addition, a summary of four ground-based investigations using one of the trainers to determine the extent to which various novel sensory stimulus conditions produce changes in compensatory eye movement responses, postural equilibrium, motion sickness svmptoms. and electrogastric responses are presented. Finally, a brief description of the general concept of dual-adapted states that underly the development of the PATs, and ongoing and tuture operational and basic research activities is presented.

A proximately 65 to $70 \%$ of crew members experience motion sickness during the first 72 hours of orbital flight on the Space Shuttle, ${ }^{1}$ and many experience spatial orientation and motion disturbances on orbit, during entry, and during the early postflight period. ${ }^{2}$ Space motion sickness (SMS) symptoms range from mild stomach awareness to frank sickness and extreme lethargy. Orientation and motion disturbances include strong sensation of "down" being other than the deck, some difficulty in rapid recognition of spatial relations between self and the visual environment. and perceptual illusions of seif or visual scene motion associated with head and body movements. ${ }^{3}$

Exposure to microgravity rearranges the relationships among signals from visual, skin, joint. muscie. and vestibular receptors. Congruence between vestibular signals and those from other receptors as well as between the vestibular otolith and semicircular canal receptors is disrupted by the absence of gravity. ${ }^{*}$ Lack of congruence among sensory signals from the spatial orientation and perceptual-motor systems leads to sensorv conflict. which may be responsible

From the NASA Johnson Space Center (Dr. Harm). Neuroscience Laboratory, Houston. Texas; and Miamı University (Or. Parker). Oxtord. Ohio. Address for reprints: Deborah L. Harm. PhD. Mall Code SD5. NASA Johnson Space Center. Houston, TX 77058. for SMS and the orientation and motion disturbances experienced by many astronauts.

People adapt to sensory stimulus rearrangements. Neurosensory adaptation is a transient or long-term modification of sensory apparatus and perceptual process that involves forming new associations between stimuli and responses to those stimuli by sensory compensation and reinterpretation. For example. adaptation to the stimulus rearrangement produced by prisms is demonstrated by motor responses, such as eye movements, as well as by sensory reactions, such as self-motion perception. ${ }^{2,4.6}$ Analogous changes are seen during adaptation to $\mathrm{mi}$ crogravity, when relationships between visual, vestibular, and somatosensory signals are altered. Adaptation is indicated by reduced subjective disturbance produced by voluntary motion after 24 to 72 hours of orbital flight as well as by perceptual and physiologic reflex changes noted during flight, entry, and immediately after landing. ${ }^{2,6-9}$

Adaptation to microgravity occurs as the result of sensory compensation or sensory reinterpretation. ${ }^{5}$ Sensory compensation occurs when the signal from one type of receptor is attenuated and signals from other receptors are augmented. In the absence of an appropriate graviceptor signal in microgravity, information from other spatial orientation receptors, such as the eyes. the vestibular semicircular canals, and the neck position receptors can be used by astro- 
nauts to maintain spatial orientation and movement control. Alternatively, signals from graviceptors may be reinterpreted by the brain. On Earth, information from the otolith graviceptors is interpreted by the brain as linear motion (translation) or tilt with respect to gravity. Because stimulation from gravity is absent during orbital flight, interpretation of graviceptor signals as tilt is inappropriate. Therefore, during adaptation to microgravity, the brain reinterprets all graviceptor output to indicate translation. This is the otolith tilt-translation reinterpretation hypothesis. ${ }^{7,10}$

Space motion sickness can be viewed as a side effect of adaptation to microgravity. Motion sickness and orientation/motion disturbances can impact crew comfort, health, safety, and performance during all phases of a mission. Therefore, treatment or prevention of these reactions is of critical importance. The primary countermeasure for SMS has been administration of anti-motion sickness medications, which may produce side effects that degrade performance. ${ }^{11.12}$ Alternatively, preadapting astronauts to the sensory rearrangements responsible for SMS and orientation disturbances may eliminate or reduce these problems.

In theory, it should be possible to preadapt astronauts to sensory rearrangements that are similar to those induced by microgravity. Development of adaptive behavioral changes and physiologic responses then should enable the astronauts to be prepared for and quickly adapt to the stimulus changes encountered in space. Eventually such adaptive responses may become reflexive, i.e., the astronaut would become "dual-adapted."

A project to develop training devices and procedures to preadapt astronauts to the stimulus rearrangements of microgravity is currently being pursued in the Neuroscience Laboratory at the NASA Johnson Space Center. Two part-task Preflight Adaptation Trainers (PATs), the tilt-translation device (TTD) and the device for orientation and motion environments (DOME) have been developed. These trainers are intended to demonstrate sensory phenomena likely to be experienced inflight and immediately postflight, allow astronauts to train preflight in an altered sensory environment, alter sensorimotor reflexes, and eliminate or reduce SMS and orientation and motion disturbances. ${ }^{*}$

\section{DESIGN PRINCIPLES FOR PATS}

Development of trainers to simulate the stimulus rearrangement of microgravity can be approached in two ways: (1) graviceptor stabilization or suppression to evoke sensory compensation, and (2) graviceptor- visual rearrangement to evoke sensory reinterpretation. Adaptation to a novel sensory environment is enhanced by active exploratory behavior in a new environment with resulting feedback and the formation of associations between sensory inputs and response outputs that promote appropriate orientation and movement in the new environment. Therefore. it is important that training devices incorporate features that support active inputs by the trainee and appropriate feedback.

The DOME-PAT is designed to achieve graviceptor stabilization. In addition to providing for graviceptor stabilization, the trainer includes features that support or enhance perceived self-motion (in 6 degrees-of-freedom) through the visual environment, with appropriate feedback for the altered/novel sensory conditions presented. Although gravity cannot be eliminated on Earth, its contribution to spatial orientation in the simulated environment can be negated. Active trainee-controlled whole body rotation and voluntary head movements about an earth-vertical axis are required to preserve the normal relationship between rotation of the visual field (in one plane at a time) and semicircular canal outputs, while keeping the trainee's orientation fixed with respect to the gravity vector. The astronaut can learn to compensate for the absence of gravity signals from the otoliths by increasing reliance on vision, semicircular canal inputs, and neck position information for spatial orientation and movement control. Virtual motion, simulated by means of a moving visual scene, with 6 degrees-of-freedom (DOF) of movement of the trainee's virtual eyepoint position through the simulated visual environment and a wide field-of-view synchronized with the actual rotation of the trainee and the projectors, supports perceived self-motion. In other words, the trainee can "be in" and "move through" visual environments in a manner not ordinarily possible on earth while keeping the gravity vector constant.

The TTD-PAT is designed to produce either the graviceptor-visual rearrangement, which is based on the previously described otolith tilt-translation reinterpretation hypothesis and the concept of "otolith ambiguity." or graviceptor suppression. Otolith receptors are stimulated both by accelerated translational motion and by tilt (pitch and roll) with respect to gravity. Because of the equivalence of linear acceleration and gravity noted by Einstein, graviceptors including otolith receptors are unable to distinguish tilt with respect to gravity from accelerated translational motion. ${ }^{13}$ An astronaut can be taught to reinterpret graviceptor output provoked by head tilts by providing movement of the visual surround that would be associated with those graviceptor re- 
sponses in microgravity. Graviceptor-visual rearrangement can be achieved by providing a tilt signal to the otolith graviceptors coupled with correlated visual scene translation, whereas graviceptor suppression is achieved by providing tilt coupled with uncorrelated visual scene translation. Separate control over the tilt and visual surround motion is a necessary feature of this system.

\section{DESIGN IMPLEMENTATION}

\section{DOME-PAT}

This device is a $3.7 \mathrm{~m}$ (12 ft) diameter spherical dome, with a $1.8 \mathrm{~m}(6 \mathrm{ft})$ diameter hole in the bottom (Figure 1). The inner surface is painted white and serves as a projection surface for two Triuniplex video projectors with custom wide-angle optics. The projectors, along with an adjustable trainee restraint assembly, are mounted on a $1.8 \mathrm{~m}(6 \mathrm{ft})$ diameter rotating base that fills the hole in the bottom of the dome. The base rides on a $92 \mathrm{~cm}$ diameter bearing, and is gear driven by a servomotor to allow computer-controlled rotation of the trainee and the projectors about a vertical axis, with angular velocities up to $120^{\circ}$ / second and accelerations up to $200^{\circ}$ second. ${ }^{2}$ This single DOF of rotation is the only real movement allowed by the system. The trainee restraint adjusts for positioning the trainee to (1) sit upright. (2) lie on either the left or right side, or (3) lie supine. For the first two positions, the projectors' optical axes are horizontal. and for (3) the projector assembly is titled back so that the images are pro-

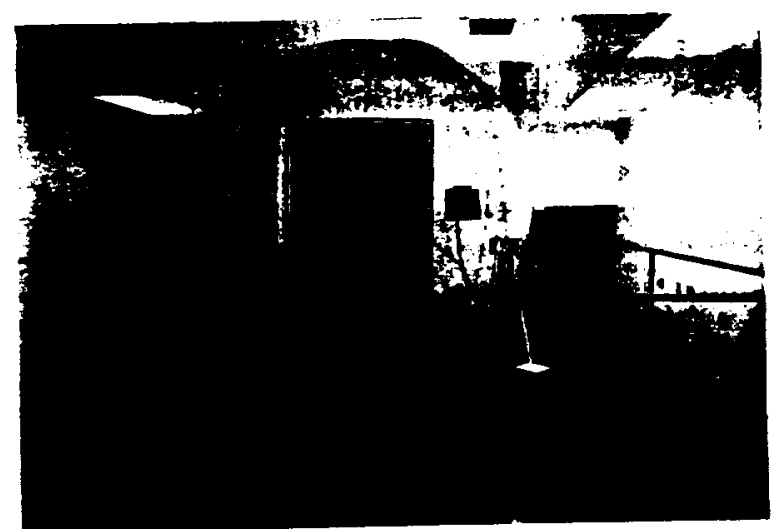

Figure 1. The DOME system consists of a large spherical dome positioned over a rotating platform on which interchangeable positioned over a rotating platform on which inters restraint svstems and two proiectors are mounted. Full trainee restraint systems and two proiectors are mounted. Full iected on the interior surtace of the dome, and a hand controller illows the trainee to move virtually through the visuai emironment. See text for detailed description. jected on the top of the dome. The field of view for the trainee is $100^{\circ} \times 170^{\circ}$, with $0.1^{\circ}$ between adjacent pixels or scan lines. This provides a very wide field of view with moderate to coarse resolution. A 6 DOF isometric joystick or a force plate may be used for interactive control by the trainee. The instructor has an identical joystick and may override the trainee whenever interactive trainee control is not desired, or when providing actual instruction to the trainee. Torque sensors in the trainee head restraint system also measure attempted head movements about nonvertical axes, and this information may be used, when appropriate, to control virtual movement of the trainee. Software allows different DOF of movement to be enabled, and provides for operator selection of different transformation of the control outputs for determining movement responses.

The database is polygons representing the visible surfaces in the interior of a closed environment. This is unlike the usual databases for aircraft flight simulators, which only represent the outside surfaces of objects. Any real or imaginary visual environment may be represented, and the operator may select different environments for different training protocols. The interpretation of trainee controls also may be modified as desired with the software to change the manner in which the controls "feel" or to limit the number of DOF of virtual movements (of the evepoint). The trainee can be placed inside a closed visual environment that represents the Space Shuttle middeck/flight deck. Spacelab, or space station. BV allowing a combination of real (rotation) and virtual motion, adequate vection should be produced to assist the trainee in the development of sensory processing algorithms that can be called on when he or she experiences similar sensory inputs in actual spaceflight.

\section{TTD-PAT}

This device is a 1-DOF tilting platform on which the subject is restrained in a car seat (Figure 2a, b). In the pitch configuration. the axis of tilt rotation is approximately aligned with the subject's interaural axis, whereas in the roll configuration the axis of rotation is approximately aligned with the subject's naso-occipital axis. A visual surround mounted on the platform moves linearly parallel to the subject's $X$ body axis in the pitch configuration and to the subject's $Y$ body axis in the roll configuration to provide a translating visual stimulus. Hence, in the pitch configuration, the subject faces the end wall and the surround translates toward and away from the subject; in the roll configuration the subject faces the side wall and the surround translates left and right of the subject. 

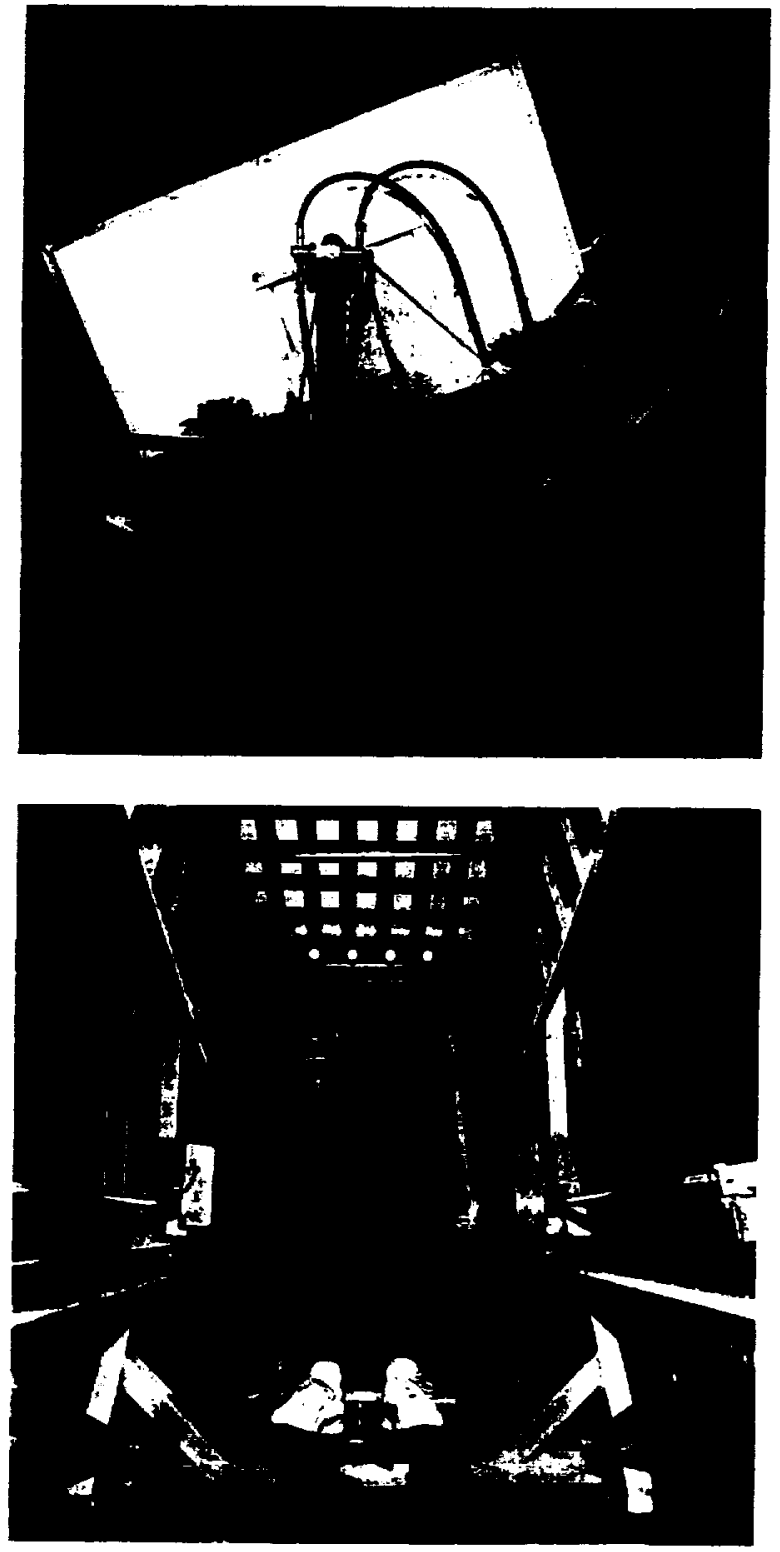

Figure 2. (a) Outside view of the Tilt-Translation Device (TTD). A one degree-of-freedom tilting platform on which are mounted a visual surround (enclosed boxl and a subject restraint. The visual surround moves linearly parallel to the subject's $X$ or $Y$ bodv axis in the pitch or roll configuration. respectively. (b) Inside view of the TTD. See text for detailed description.

The visual surround is a $2.74 \mathrm{~m} \times 0.89 \mathrm{~m} \times 0.91 \mathrm{~m}$ (approximately $9^{\prime} \times 3^{\prime} \times 3$ ) white box with three-dimensional vertical black stripes on the inside walls and horizontal stripes on the ceiling. Four successively smaller outlined black squares and a solid black square in the center are attached to the inside of the end walls. The line width and separation between lines is progressively smaller from the outer to the inner square to produce the appearance of a tunnel. This "tunnel" effect produces a visual stimulus distance ambiguity that is designed to allow the subject to scale perceived distance to the walls so that the expanding and contracting optic flow and looming pattern matches the simulated physical acceleration stimulus provided by the tilting base. ${ }^{14}$ In addition, the linear translation of the visual surround is intended/designed to elicit linear vection (self-motion). The amplitude, frequency, phase, and waveform shape of the tilt base and surround translation are independently controlled by a microcomputer.

As described earlier, the otoliths' response to tilt is indistinguishable from their response to linear acceleration. Similarly, somatosensory/tactile/touch and pressure receptor responses to tilt from the back. buttocks, and posterior thighs can be interpreted as either tilt or linear acceleration. Given our current understanding of otolith and somatosensory ambiguity, and the contributions / role of visual patterns and optic flow to the perception of orientation and selfmotion, the task becomes one of determining how to arrange the relationship among the visual, otolith, and somatosensory stimuli in a way that biases the central nervous system (CNS) to interpret the combined sensory signals as linear self-motion.

The TTD-PAT has been used extensively in ground-based investigations to determine the extent to which graviceptor-visual rearrangement stimulus conditions produce changes in eye movement responses, ${ }^{15.16}$ postural equilibrium, ${ }^{17}$ and motion sickness symptoms similar to those observed in astronauts. An overview of four ground-based investigations designed to examine the effect of exposure to TTD-PAT stimulus conditions on: (1) compensatory vertical eye movement gain (CVEMG), (2) vertical net gaze compensation, (3) postural equilibrium, and (4) motion sickness symptoms and electrogastrogram (EGG) responses, is presented next.

\section{METHOD}

\section{Motion Profile}

In all of the experiments described here, the displacement amplitude of surround translation was \pm 15 inches, and a $270^{\circ}$ phase relation between tilt and surround motion was employed (Figure 3). Based on a set of ground-based investigations designed to examine perceptual responses to a variety of visual surround translation and tilt motion profiles, we determined that a $270^{\circ}$ phase relation between tilt and surround motion would best support the perception 


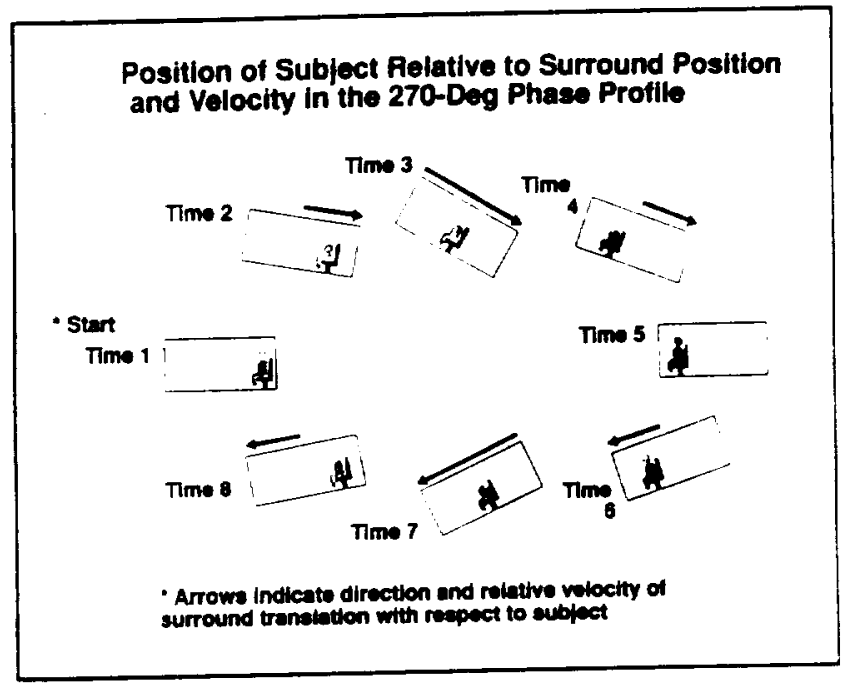

Figure 3. Position of subject relative to visual-surround position and velocity for the $270^{\circ}$ phase stimulus rearrangement condition. At time 1. the visual surround is at its most forward position and is stationary with respect to the subject: the subject is uprignt. At time 2. the surround has moved rearward toward the subject and both the subiect and the surround are pitched backward. As indicated the subject and the surround are pitched backward $i 1^{0}$ in of its peak velocity at this time. At time 3 , the visual surround reaches peak velocity and the subject is in the most backward pitch position. At time 4 , the subject and surround are returning to upright and the surround velocity is reduced. Finally, at time 5 . the subiect is upright and the surround is stationary. Forward transiation of the surround with respect to the subject during forward pitch of both the surround and the subject occurs from time 6 to time 1 .

of linear self-motion. ${ }^{18}$ The rationale for this phase relation is as follows: During "real" translation. when an individual is upright. maximum forward acceleration produces maximum pressure on an individual's back and maximum rearward (occipital) displacement of the otoconia. The $270^{\circ}$ phase motion profile in the TTD mimics the stimulus conditions of "real" translation, i.e.. stimulation of maximum for- ward translation in the TTD occurs when maximum rearward tilt at $270^{\circ}$ is coupled with maximum rearward visual scene velocity. The translational selfmotion cues associated with the visual surround velocity are congruent in time and direction with graviceptor force and pressure cues associated with the pitch position (Figure 3). The other characteristics of the motion profile, subjects $(\mathrm{N})$, and number and duration of exposures for each experiment are presented in Table I.

\section{Experiment 1: Compensatory Vertical Eye Movements}

Hypothesis. Under normal conditions with the eyes open. compensatory vertical eye movement gain is approximately unity; i.e.. the eves move in an equal and opposite direction to head tilt. If exposure to the $270^{\circ}$ phase relation between tilt and surround motion stimulus rearrangement (SR-270) leads to reinterpretation of tilt as linear translation, then tilt in darkness (No-SR) after exposure to this motion profile should result in decreased vertibular-induced compensatory eye movement gain.

Procedures. Standard electrooculography (EOG) techniques using infant electrodes positioned above and below the right eye and the ground electrode behind the left ear were used to record CVEMG. Control and exposure sessions were separated by 1 week, and the order was randomized. The control session (No-SR) consisted of tilt only in darkness at the same frequency and tilt amplitude and for the same duration as the adaptation exposure (SR-270). Compensatory vertical eye movement gain was recorded in darkness with the eyes open before exposure. after 30 minutes. and after 45 minutes of exposure while the subject attempted to fixate a floor-fixed imaginary target during sinusoidal tilt.

TABLE I

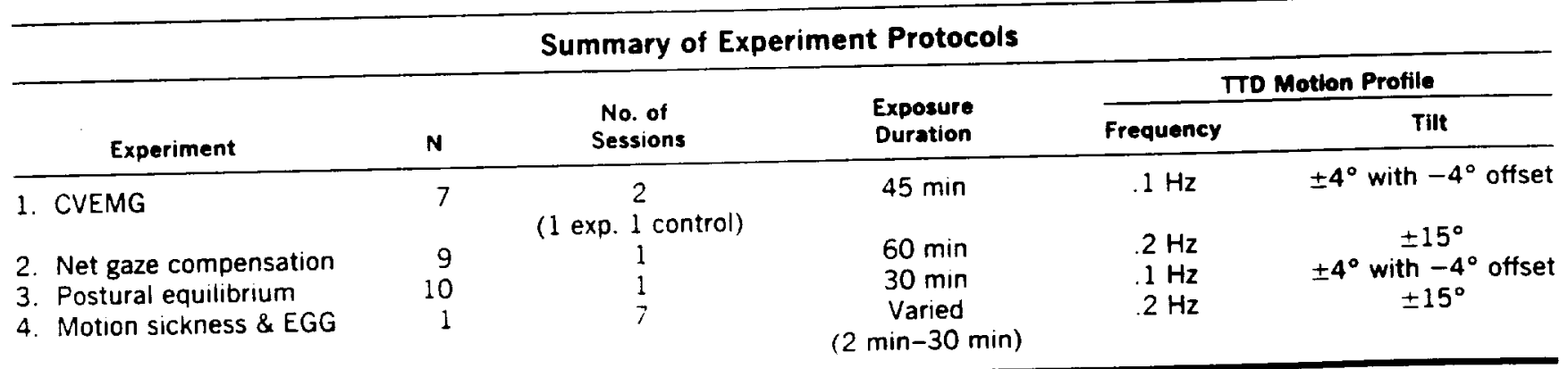


Results. Compensatory vertical eye movement gain was defined as vertical eye movement amplitude divided by head pitch amplitude. Eye movement gain values were analyzed using a two-way (conditions by time) repeated measures analysis of variance. The predicted conditions by time interaction illustrated in Figure 4 was significant $[F(2,11)=22.16$; $<0.0001]$. The simple main effect of time was significant for the SR-270 condition $[F(212)=26.63 ; P$ $<.0001]$. Post hoc testing indicated that the pre-exposure gain was greater than the postexposure gain at both 30 and 45 minutes, and that the gain values 30 and 45 minutes after exposure were not different. The simple main effect of time was not statistically significant for the No-SR condition.

\section{Experiment 2: Vertical Net Gaze Compensation}

Hypothesis. Under normal conditions, stable gaze (ability to maintain fixation on a visual target) is maintained through a combination of slow-phase compensatory eye movements and compensatory saccadic eve movements (net compensation). As for experiment 1, we hypothesized that exposure to the SR-270 stimulus condition would result in reduced CVEMG during head pitch. This gain reduction would result in "undershooting" during a gaze fixation task: the eve movement should be of insufficient amplitude to compensate for real head pitch when the subject attempted to maintain fixation on an imagined earth-fixed target.

Procedures. Electrooculogram recording techniques were identical to those of experiment 1 . Before and

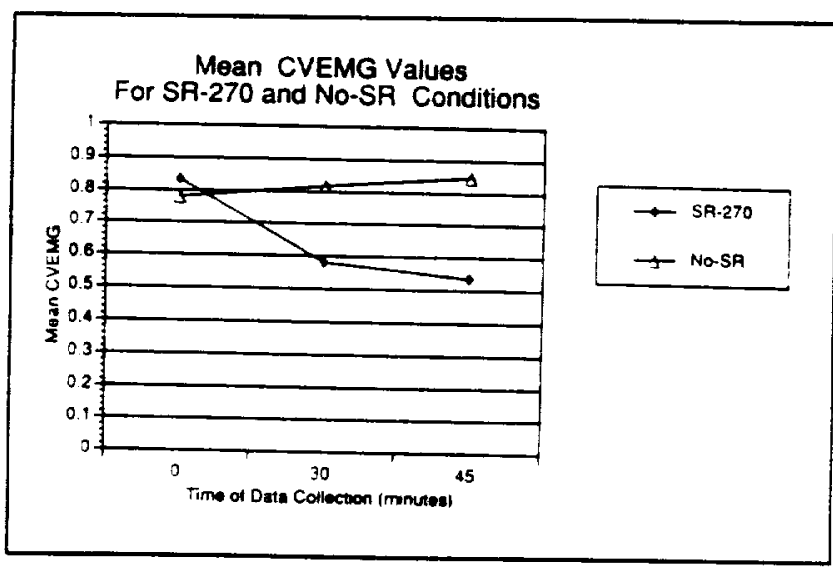

Figure 4. Mean compensatory vertical we movement gan (ClEMG) values as a function of time of testing. CVEMG vilues atter 30 and 45 minutes of exposure to the $270^{\circ}$ motion pronle (SR-2ZO) are compared with vaiues oblained before and after exposure 10 tult in darkness $(\mathrm{No}$-SR!

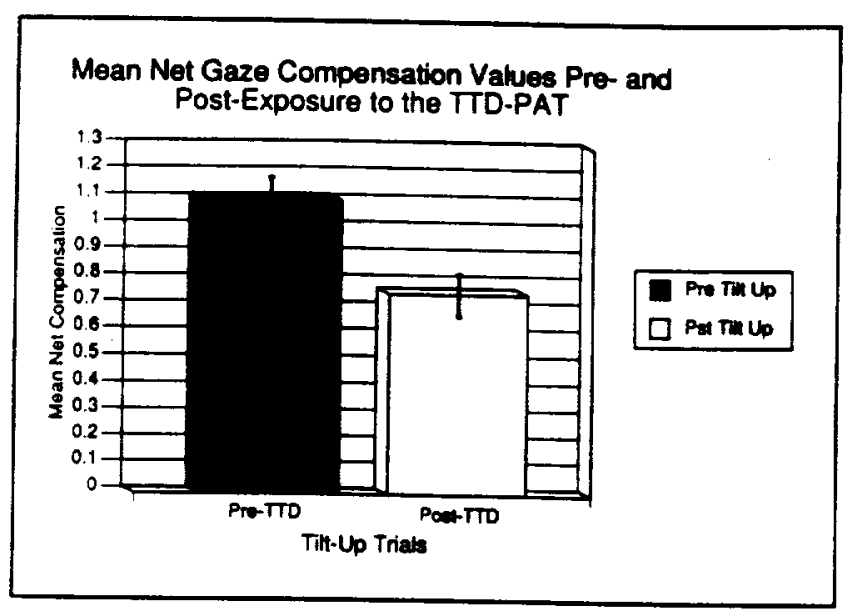

Figure 5. Mean net gaze compensation values for pitch tilt up trials as a function of time of testing (pre-TTD exposure versus post-1 hour of $270^{\circ}$ TTD exposure).

after 60 minutes of TTD exposure, subjects completed 20 trials of a gaze task (10 trials each of $\pm 15^{\circ}$ pitch tilt). The subject fixated a floor-fixed lightemitting diode (LED) in the lighted surround. One second before the chair tilting, all lights were extinguished and the subject was instructed to maintain fixation at the remembered position of the LED until the chair tilt stopped and the LED was turned on again. The subject refixated the LED as quickly as possible if he or she was off target at this time.

Results. Net compensation may be defined as the sum of saccadic and slow phase compensatory eye movements. Net compensation values were determined by the following formula: Net compensation $=B / A$. where $B=$ the amplitude of compensatory vertical eye movements during chair tilt (slow phase + saccadic) and $A=$ the amplitude of the difference between initial eye position and final eye position after any correction or refixation saccade. Net compensation values $=1$ indicate perfect compensation. values $<1$ indicate undershoot, and values $>1$ indicate overshoot. Data from the tilt-up $\left(-15^{\circ}\right)$ trials only are presented here. Pre-TTD to post-TTD net gaze compensation values were analyzed using a Student $t$ test. The predicted decrease in mean (averaged over all 10 trials) net gaze compensation illustrated in Figure 5 was significant $[\mathrm{t}(8)=2.603 ; \mathrm{P}$ $<.035]$. Subjects tended to undershoot the target, requiring a saccadic eye movement to refixate the target. Although not shown here. inspection of the net compensation values across post-TTD trials indicated a fairly rapid decay of the adaptation effect. When only the first four tilt-up post-TTD trials were analyzed, the level of statistical significance was 
greater than when all 10 trials were analyzed $[t(8)$ $=3.264 ; P<.012]$. The magnitude of pre-to post-TTD net gaze compensation for the tilt-down $\left(+15^{\circ}\right)$ trials was only slightly smaller than that observed in the tilt-up trials; and, the response decay was also similar to that observed in the tilt-up trials.

\section{Experiment 3: Dynamic Postural Equilibrium}

Hypothesis. Maintenance of postural equilibrium is achieved through appropriate integration of visual. vestibular, and proprioceptive (primarily ankle) signals. If tilt is reinterpreted as translational motion after exposure to the TTD profile described earlier. decreases in postural equilibrium should occur under conditions in which the CNS is forced to rely on vestibular signals for postural control, i.e.. where visual signals are eliminated or altered and ankle proprioceptive signals are eliminated.

Procedures. Postural equilibrium was assessed preand post-TTD exposure using a modified commercial dynamic posturography system (EquiTest-Neurocomj. Subjects were exposed to a series of six sensory organization tests in which their ability to maintain stable upright posture was assessed under normal and reduced/altered sensory feedback conditions. Each sensory organization test was presented three times for a 20-second period during each trial. Data from sensory condition six (SC-6) only are presented here. In SC-6, illustrated in Figure 6. both the visual

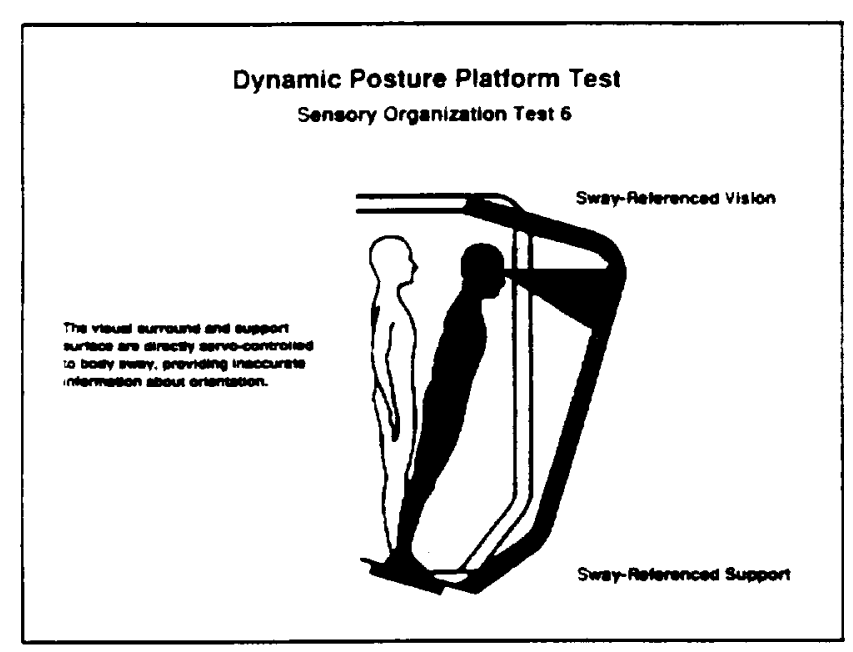

Figure b. Sensorv organization test 6 presented witn the Veurocom Equitest dynamic posturography system. The visuai surround and support base are swav reterenced: ankle proprioceptwe treaback is

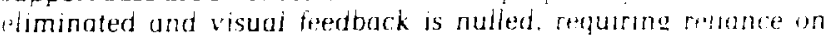
restibular teedback for postural control surround and the support base are sway-referenced. In this condition, ankle proprioceptive feedback is eliminated and visual feedback is nulled, requiring reliance on vestibular feedback for postural control.

Results. Postural equilibrium scores are a measure of overall stability and are based on the peak-to-peak anterior-posterior (A-P) center of gravity sway during the 20-second trial:

$$
\text { Equilibrium Score }=\left[1-\frac{P-P \text { sway }}{12.5}\right] \times 100
$$

where 12.5 is the maximum stable sway amplitude expected in a normal population. The predicted preto post-TTD exposure decrease $(63.9-44.9)$ in postural equilibrium. illustrated in Figure 7, was significant (t $[9]=3.785 ; P<.005$ ). Decreases in SC-6 postural equilibrium scores of astronauts $(n=14)$ preflight to postflight are also illustrated in Figure 8 for comparison with pre- to post-TTD findings. The magnitude of the decrease in postural equilibrium within 2 to 3 hours postflight is only slightly larger than that observed after a 30 -minute exposure to the TTD.

\section{Experiment 4: EGG and Motion Sickness Symptoms}

Hypothesis. The normal basic electrical rhythm of the stomach is approximately 3 cycles per minute (cpm). Tachygastria (4-9 cpm) is often observed immediately before and during nausea. ${ }^{17,19}$ If repeated exposure to motion sickness provoking stimulus conditions leads to adaptation, then the severity of gastric symptoms should be reduced over time. Tachygastric electrical rhythms should accompany nausea and return to the normal gastric basic electrical rhythm as subjective gastric symptoms subside.

Procedures. Seven subjects were exposed to the TTD six times. The exposure sequence was as follows: days $1,3,5,9,17$. and 33 ; an interval of 48 hours separated the first three exposures with an exponential increase in the time interval between each of the remaining exposures. Net gaze compensation, perceptual responses, and motion sickness symptoms were monitored throughout all test sessions. Three subjects developed sufficient motion sickness symptoms within the first few minutes of the first exposure that the test had to be terminated. Two of the three motion sick subjects reported no symptoms during any of the remaining five sessions. The other motion sick subject. whose data will be presented here. was extremely susceptible to the TTD motion protile. Because of the severity of motion sickness 


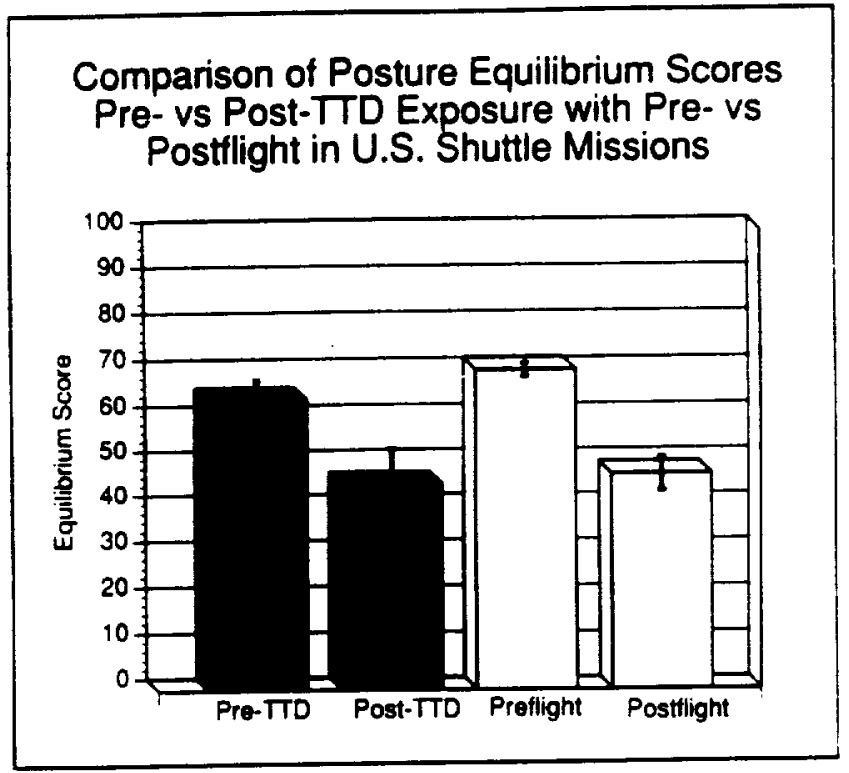

Figure 7. Mean postural equilibrium scores for sensory organization test 6 as a function of time of testing (before versus after 30 minutes' exposure to the $270^{\circ}$ TTD motion profilel compared with mean postural equilibrium scores for sensory organization test 6 iss a function of 5 to 8 days exposure to microgravity during $L . S$. Shuttle missions (betore versus after flight).

symptoms, we initiated EGG measurements beginning on test day 5 (his third session) to assess changes in gastric electrical rhythms associated with nausea. Three electrodes were placed on the subject's abdomen: one was positioned along the left midclavicular line 1 to 2 inches below the costochondral margin. one was positioned at the midpoint between the $\mathrm{xy}$ phoid process and the umbilicus, and the ground was placed on the rib cage of the subject's left side (Figure 8).

Results. The EGG signal was sampled at $120 \mathrm{~Hz}$. Because of the presence of motion artifact and electrode drift, running spectral analysis of the data was not possible. Instead, segments without obvious motion artifact and with only monotonic drift were selected as close to the time period of interest as possible (the last minute of the baseline and the exposure periods). Each segment was 8192 points in length (1.14 minutes) and was low-pass filtered using a 35 point Chebyshev window with a $40-\mathrm{dB}$ sidelobe rolloff and a $0.4-\mathrm{Hz}$ cutoff. Segments were then linearly detrended and Hamming windowed before power spectral density calculations using a fast Fourier Transform. The densities for the first two bins ( 0 and $0.015 \mathrm{~Hz}$ ) were set to zero, effectively highpass filtering the data. The power for the average baseline was calculated as the average density of each frequency bin for days 5,17 , and 33 (the baseline data for day 9 were not usable).

The data are presented in purely descriptive terms. Tolerance time to the TTD stimulus conditions gradually increased over the test sessions from 2 minutes on day 1 to 30 minutes by day 9 . Motion sickness symptoms gradually decreased from facial flushing, increased perceived body warmth, and severe nausea on day 1 to slight sensation of body warmth, mild sweating, and stomach awareness by day 17 . Although the subject tolerated the entire 30-minute exposure to the TTD on day 33, he reported more symptoms than on day 17. Finally, EGG illustrated in Figure 9 shows the highest power in the average baseline data was within the normal basic electrical rhythm frequency range $(2.5-3.5$ cycles/ $\mathrm{min}$. An increase in the power density in the tachygastric frequency range of 4 to $9 \mathrm{cpm}$ was clearly evident on day 5 . On day 9 , some tachygastria was still evident, but the power density was less than that observed on day 5 , and there was an increase in the power density for the normal gastric electrical frequency range of 2.5 to $3.5 \mathrm{cpm}$. By day 17 , tachygastric activity was essentially absent, and the highest power was within the normal basic electrical rhythm frequency range. On day 33 , the gastric elec-

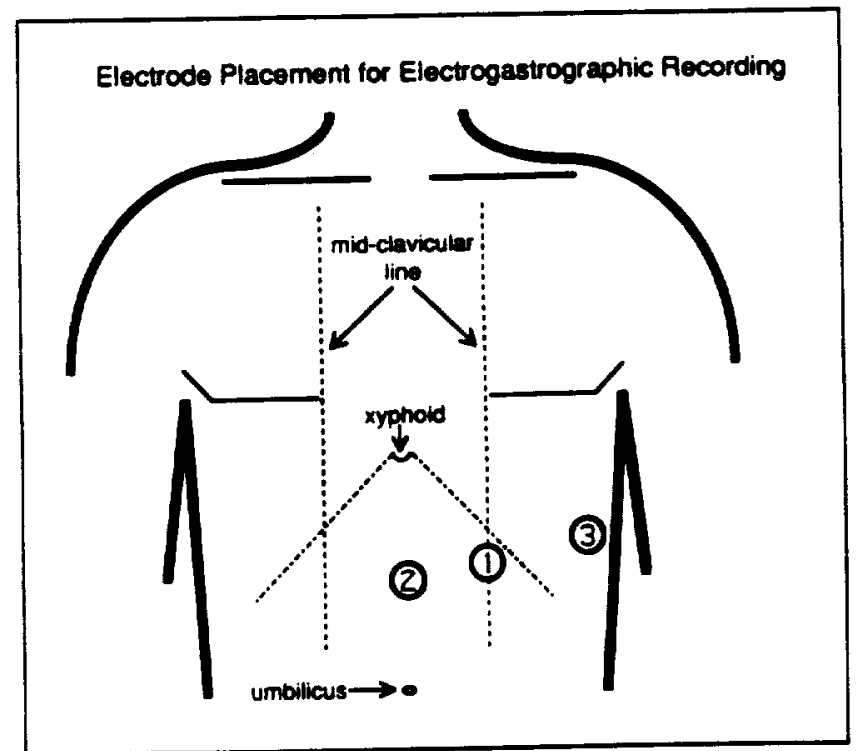

Figure 8. Electrode placement for electrogastrographic (EGG) recording. Electrode 1 is positioned along the left midclavicular line $i$ to 2 in. below the costochondral margin; electrode 2 is positioned at the midpoint between the xyphoid process and the umbilicus; the ground electrode ${ }^{3}$ is positioned on the lower left lateral rib rage. 


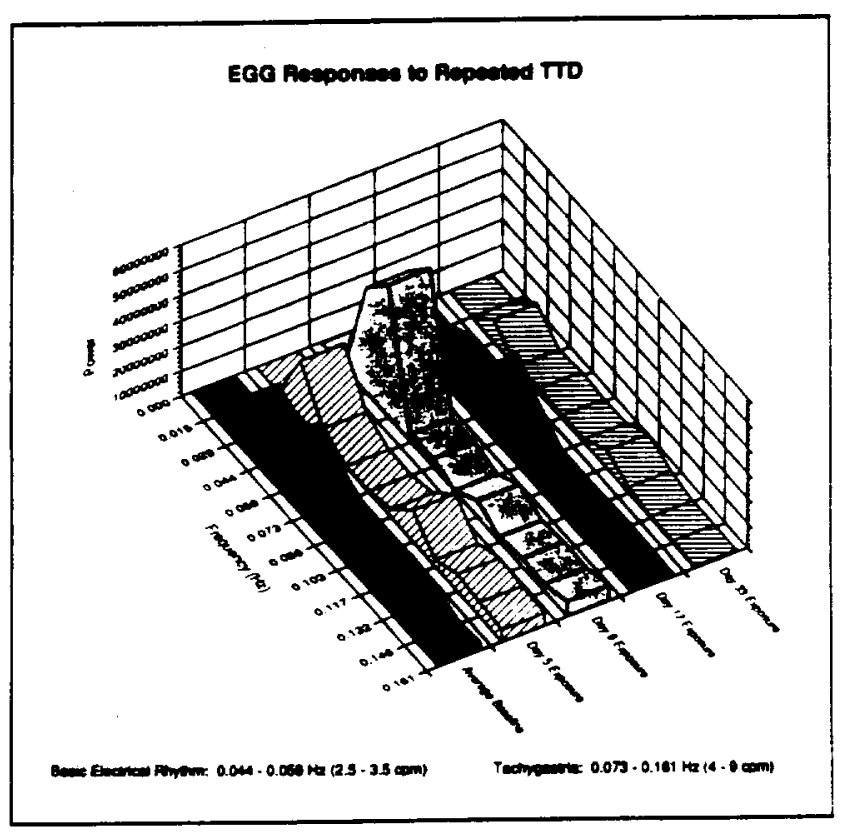

Figure 9. Power spectral density plot of EGG responses during the last minute of baseline recording (average of davs 5,17 , and 33 . and the last minute of exposure to the $270^{\circ}$ TTD motion protile on davs $5,9,17$, and 33 .

trical rhythm again showed an increase in the tachygastria range but to a lesser extent than on days 5 and 9 . This would suggest that the adaptive responses began to decay when the interval between exposures to the TTD was extended to 2 weeks.

\section{CONCLUSIONS FROM TTD EXPERIMENTS}

Exposure to the $270^{\circ}$ TTD stimulus rearrangement results in significant decreases in CVEMG. vertical net gaze compensation, and postural stability. After repeated exposures, an individual who was initially highly susceptible to the stimulus conditions gradually adapted in terms of tolerance time, reduction in the number and severity of motion sickness symptoms and return to normal EGG rhythm. This individual showed evidence of retention of adaptive responses even with increasing intervals between sessions.

The results were consistent with the otolith tilttranslation reinterpretation model and the hypothesis that the $270^{\circ}$ phase relation between tilt and surround motion in the TTD leads to reinterpretation of otolith signals as linear self-motion. The general schedule of repeated exposures used in experiment 4 shows promise in establishing adaptive responses and increasing the time of retention of the responses. Development of optimal training sched- ules for preadapting Shuttle astronauts is an essential component of the overall effort to preadapt crew members to the sensory conditions of microgravity.

\section{DISCUSSION}

The general concept underlying the development of PATs is that the trainee can learn to "recalibrate" the relationships between vestibular, visual, and somatosensory signals in a manner that would be appropriate to microgravity. It is hypothesized that after training in these devices, compensatory eye movement responses, postural muscle reflexes, and self-motion and orientation experiences in relation to visual scene movements and position, would be appropriate to the weightless-adapted state. In other words, new sensory and sensorimotor programs appropriate for microgravity would be developed. The available data in support of this hypothesis are largely derived from our investigations with the TTD device. Similar investigations will need to be carried out, and are being planned for the DOME-PAT system. This system is relatively new and is still undergoing some hardware and software modifications. The DOME is currently being used to demonstrate perceptual illusory phenomena to astronauts and to teach crew members to describe their perceptual experiences. Based on comments from a limited number of astronaut participants, a number perceptual experiences produced by the DOME system are similar to those experienced inflight, during entry, or immediately postflight.

Adaptive responses that are appropriate for one set of sensory stimulus conditions, such as those present in microgravity, are inappropriate for the normal $1 \mathrm{~g}$ environment on earth. Return to earth's gravitoinertial environment requires a period of readaptation. Readaptation is the process or processes by which the trainee relearns the associations between sensory inputs and response outputs that promote appropriate spatial orientation, stable vision, posture and locomotion on earth. Because the central nervous system is "plastic," people are able to learn and store perceptual, sensory and sensorimotor responses appropriate to different sensory stimulus conditions, and they can learn to invoke these alternative responses almost immediately when the conditions change. For example, experienced SCUBA divers exhibit altered compensatory eye movement gains immediately on donning their diving masks. Apparently, they have learned that a higher gain is required to compensate for the magnification of the visual scene due to the air-water interface. Removal of the mask is associated with a nearly immediate return of the normal gain. Similarly, development of 
optimal training schedules should allow astronauts to develop dual-adapted states, one appropriate for the normal earth environment and one for microgravity, and to develop the ability to switch rapidly between these states.

Currently, PAT development and evaluation are proceeding along two paths: an "operational" path and a "basic research" path. Some of the activities being pursued along the operational path include: (1) "fine tuning" the sensory stimulus conditions produced by both PAT devices to correspond with astronaut's descriptions of their perceptual experiences during different phases of their Shuttle mission. (2) developing optimal training schedules. (3) developing multimedia education and training materials. and (4) developing appropriate training scenarios. Activities pursued along the basic research path include: (1) concept verification. (2) testing of the otolith tilt-translation reinterpretation hypothesis, and (3) identification of reliable dependent measures of sensory, sensorimotor, and perceptual responses to altered sensory conditions. Over the next few years we expect to continue the "fine tuning" of stimulus conditions and training procedures and begin to evaluate the effectiveness of various components of the training with an astronaut population.

The research presented in this paper could not have been accomplished without the dedication and support of many staff members in the Johnson Space Center Neuroscience Laboratory. In particular. the authors would like to thank Noel Skinner. Gwen Sandoz. Bill Becker, Denine Doxey, Kathy Duncan. Bill Worthington. Jenny Krug. Freddie Ferrara. Bill Crosier. and Drs. Reschke. Bloomberg. Paloski and Kennedy. A special thanks to Elisa Allen and Jill Kuennen for their assistance in the preparation of this manuscript.

\section{REFERENCES}

1. Davis IR. Vanderploeg IM. Santy PA. Jennings RT. Stewart DF: Space motion sickness during 24 flights of the Space Shuttle. Av'iat Space Environ Med 1988;38:1185-1189.

2. Reschke MF. Parker DE: Effects of prolonged weightlessness on self-motion perception and eye movements evoked by roil and pitch. Aviat Space Environ Med 1987:58:A153-158.

3. Harm DL. Parker DE: Perceived self-orientation and self-motion in weightlessness. after landing and during preflight adapta. tion training. Irn Vest Res 1993 (in press).
4. Parker DE. Parker KL: Adaptation to the simulated rearrangement of weightlessness, in: Crampton GH (ed.): Motion and Space Sickness. Boca Raton, FL: CRC Press, 1990;247-262.

5. Parker DE: Human vestibular function in weightlessness. / Clin Pharmacol 1991:31:904-910.

6. Paloski WH. Reschke MF. Black FO, Doxey DD, Harm DL: Recovery of postural equilibrium control following space flight. Ann N Y Acad Sci 1992;656:747-754.

7. Parker DE. Reschke MF. Arrott AP, Homick JL, Lichtenberg BK: Otolith tilt-translation reinterpretation following prolonged weightlessness: Implications for preflight training. Aviat Space Environ Med 1985;56:601-606.

8. Bloomberg JJ, Reschke MF, Harm DL, Michaud LI, Kulecz WB Peters BT: Head and gaze stability during locomotion: I. Analysis of linear head movement. Aviat Space Environ Med 1991:62:477. 9. Paloski WH. Harm DL, Reschke MF. Doxey DD, Michaud LI. Parker DE: Postural changes following sensory reinterpretation as an analog to space flight. Proceedings of the Fourth European Symposium on Life Science Research in Space 1990:175-178.

10. Young LR. Oman CM, Watt DGD, Money KE, Lichtenberg BK: Spatial orientation in weightlessness and readaptation to Earth's gravity. Science 1984:225:205-208.

11. Wood CD, Manno JE, Wood MJ, Manno BR, Redetzki HM: Mechanisms of antimotion sickness drugs. Aviat Space Environ Med 1987:58:A262-265.

12. Kennedy RS. Odenheimer RC, Baltzley DR, Dunlap WP. Wood CD: Differential effects of scopolamine and amphetamine on microcomputer-based performance tests. Aviat Space Environ Hed 1990:61:615-621.

13. Mayne R: A systems concept of the vestibular organs, in Kornhuber $\mathrm{H}$ (ed.): Vestibular System Part 2: Psychophysics. Applied Aspects and General Interpretations. Berlin: Springer Verlag 1974:493-580.

14. Regan D, Beverley KI: Visually guided motion: Psychophysical evidence for neural mechanism sensitive to flow patterns. Science 1978: 205:311-313.

15. Harm DL. Boomberg JI, Reschke MF. Skinner NC, Liao CA, Michaud LI. Parker DE: Adaptive modification of gaze control following tilt reinterpretation. Aviat Space Environ Med 1991: $62: 477$.

16. Michaud L. Parker DE. Harm DL: Changes in the vestibuloocular rellex associated with simulated stimulus conditions of space flight. Proceedings of the Annual Conference of the Society for Neuroscience 1989:514.

17. Stern RM. Koch KL. Leibowitz HW, Lindbland IM. Shupert CL. Stewart WR: Tachygastria and motion sickness. Aviat Space Environ Med 1985;56:1074.

18. Reschke MF, Parker DE. Harm DL. Michaud L: Ground-based training tor the stimulus rearrangement encountered during space flight. Acta Otolaryngol (Stockh) Suppl. 1988:460:87-93.

19. Hu S. Grandt WF, Stern RM. Koch KL: Motion sickness severity and physiological correlates during repeated exposures to a rotating drum. Aviat Space Environ Med 1991;62:308-314. 